\title{
An Intelligent Fire-Detection Method Based on Image Processing
}

\author{
Mingxiu Lin ${ }^{1, a}$, Wenlin Chen ${ }^{2, b}$ Baosong Liu $^{3 c}$ and Lina Hao ${ }^{3, d}$ \\ ${ }^{1}$ College of Information Science and Engineering, Northeastern University ,Shenyang, China \\ ${ }^{2}$ College of Resources and Civil Engineering, Northeastern University ,Shenyang, China \\ ${ }^{3}$ School of Mechanical Engineering and Automation, Northeastern University, Shenyang, China \\ alinmingxiu@ise.neu.edu.cn, bchenwenling@neu.edu.cn, ${ }^{c}$ liubaosong1987@126.com \\ ${ }^{d}$ haolina@me.neu.edu.cn
}

Keywords: image sequence, fire-detection, time difference, color model, shape features

\begin{abstract}
How to explore higher efficient and more credible fire-detection system by rapid development of computer and image processing techniques has aroused public's extensive attention. To achieve fully automatic surveillance of fires, an intelligent fire detection method based on a multi-stage decision strategy of image processing is proposed. Both static and dynamic characteristics of the fire images sequence are considered. First of all time difference is used to process the gray-scale image to obtain the moving region in the scene, secondly apply color segmentation to get the ROI of fire region, thirdly shape features such as randomness of area size, edge likelihood are calculated to avoid some interference, at last the polygonal and irregular characters of flame like sharp corners and circularity are used to identified the fire. Experimental result shows the Fire-detection method presented in this paper could detect fire in the image sequence effectively, and it is capable of distinguishing Environmental light changes, background color interference and light false identification. Multi-stage decision strategy can improve the algorithm performance and reduce false-alarm rate. The proposed method has broad application prospects in the important military, social security, forest-fire alarm, commercial applications, and so on.
\end{abstract}

\section{Introduction}

Fire detection becomes more and more appealing because of its important application in surveillance systems of different industry such as military, social security and economical. There are different methods for detecting fire in different features and applications [1]. Sensors are used to measure the desired parameters in most of the methods. Applying several sensors is essential in order to cover all desired area while it is not cost-effective. How to explore higher efficient and more credible fire-detection system by rapid development of computer and image processing techniques has aroused public's extensive attention.

To avoid the large scale of damage, it is necessary to have a system to discover the incident as fast as possible. Computer vision-based fire detection algorithms are usually applied in closed-circuit television surveillance scenarios with controlled background. However it is impossible to keep the human observation of Closed-Circuit Television (CCTV) for 24 hours. If the fire detection system through image processing can warn fire state automatically, it will be very convenient, and it can be possible to minimize the damage. The recent methods for fire detection are based on the color pixel recognition and motion detection, many wrong alarms are happening and this decrease the performance of the systems [2].

In this paper, to achieve fully automatic surveillance of fires, an intelligent fire detection method based on a multi-stage decision strategy of image processing is proposed. Both static and dynamic characteristics of the fire images sequence are considered. 


\section{Analysis of Fire Visual Features}

It is well known that fire has unique visual signatures. It can be divided into two types as static characteristics and dynamic characteristics, both are significant and always co-exist complementary, but also has a certain complexity and diversity, it is difficult to be precise description and modeling. The visual features chosen to decide if there is a fire has happened in the scene must be distinctive consistency and independent.

Flame static visual features include spectral information and spatial structure. The former mainly refers to the brightness and color, while the latter mainly contains the internal structure and edges of the region of the flame color pixels. Under general background illumination, the flame has outstanding brightness which is an important clue to detect the flame in gray images, and flame color range from red to yellow with a specific model of dynamic range in RGB, HIS, HSV color space[3]. Static spectral properties alone are not enough to identify the real flame. Similar scenes in the sun or other sources of flame color easily lead to false detection. The burning flame in a constant state of change determines the complexity of the regional structure. Firstly, the flame region is different from the rigid structure. It is a cloud-like deformation zone with constantly changing of the shape, size and color distribution. Secondly, the internal flame region also shows a certain hierarchy corresponding to the spectral color with temperature.

Flame dynamic features are unique and complex, the flame is random, Hierarchy and time-frequency. On one hand the flame zone moves at low frequency, which performance as regional deformation, growth and movement; on the other hand the flame edge has random movement . The former reflects changes in the state of the flame, such as the movement and deformation generated by the fire regional scale changes or air flow; the latter refers to the disorder flame flashing, that is a continuous high-frequency timing variations.

It is obvious that there are close correlations between the static characteristics and the dynamic characteristics of the flame. Both characteristics will be taken into account when modeling and detecting fire. In this paper the moving region, color segmentation, randomness of area size, edge likelihood, sharp corner and target circularity are considered to detect fire and decrease the false alarm.

\section{Fire Feature Extraction algorithm}

Moving regions detection. The detection of moving regions is a fundamental key in video fire detection [4], which is the first stage of the proposed method. In this stage the time difference is used to detect moving regions quickly. The time difference image $d(i, j)$ is calculated as follows:

$$
d(i, j)=g_{n}(i, j)-g_{0}(i, j)
$$

where $g_{n}(i, j)$ is the nth frame of the input images, $g_{0}(i, j)$ is the background image which is updated during the surveillance. Here the input color images is transformed into grayscale, Binary image $g_{\text {result }}(i, j)$ of Eq. 2 expressed the changing region in the image that refers to the moving region.

$$
g_{\text {result }}(i, j)= \begin{cases}1 & d(i, j)>T_{1} \\ 0 & d(i, j) \leq T_{1}\end{cases}
$$

Here define $g_{\text {sum }}$ as the move significant of recent image as follows:

$$
g_{\text {sum }}=\frac{\sum_{i=1}^{m} \sum_{j=1}^{n} g_{\text {result }}(i, j)}{m \times n}
$$

If $g_{\text {sum }} \geq T_{2}$, that there is a potential fire. Here $\mathrm{T}_{1}, \mathrm{~T}_{2}$ are thresholds which are picked by guesswork and experience, $T_{1}$ decide algorithm sensitivity to a single pixel change while $T_{2}$ decide algorithm sensitivity to changes in the whole image.

Color segmentation. According to most fire detection papers presented in the literature and based on our own experiments, we notice that fire has very distinct color characteristics; it is the most powerful single feature for finding fire in video sequences. The color of flame is usually between red and 
yellow [5]. In the RGB color model, it is noticed that for a given fire pixel, the value of red channel( $R)$ is greater than the green channel $(\mathrm{G})$, and the value of the green channel $(\mathrm{G})$ is greater than the value of blue channel(B), and the value of red channel(R) must be greater than a certain threshold $\left(R_{T}\right)$ which is obtained by experiment. HIS is also a useful color model; in this paper we combined RGB and HIS as follows:

$$
S>(255-R) \times S_{t} / R
$$

Where, $\mathrm{R}$ is the value of red channel of RGB model, and $\mathrm{S}$ is the Saturation of HIS model. The method can avoid objects with strong light reflections being misjudged to be flames. The threshold $S_{t}$ is obtained by a large number of experiments. Under the same detection condition, the suspicious fire area extracted by the promoted algorithm is more precise, the false alarms are reduced effectively.

Randomness of area size. In image processing, the area size represents the number of pixels of the object. Because of fire flickering, the edge will be beating and the area size of the suspicious area will change from frame to frame, but the contours are similar [3]. We calculate the Randomness of area size using the formula described below:

$$
\nabla A=\frac{A_{i}-A_{i-1}}{A_{i}}, i=1,2,3 \text {.. }
$$

where $A_{i}$ means the area size of the potential fire regions in the current frame and $A_{i-1}$ means the area size of the potential fire regions in the former frame. In case a hard decision rule is used, fire is assumed if $\nabla \mathrm{A}_{\mathrm{i}}>\lambda$, where $\lambda$ is a decision threshold.

Edge likelihood. From the geometric properties point of view, the edges of adjacent frames of flame sequence are unstable, but the overall edges have a stable similarity. "Sobel" detection operator is used to get the edges of the suspicious fire, and then use the similarity formula as Eq. 6 to calculate the edge likelihood $\varepsilon_{i}$ :

$$
\varepsilon_{i}=\frac{\sum b_{i}(x, y) \cap b_{i+1}(x, y)}{\sum b_{i}(x, y)}
$$

where $b_{i}(x, y)$ are the pixels of flame in the current frame and the $b_{i+1}(x, y)$ are the pixels of flame in the latter frame. During the fire detection a threshold $\theta$ should be chosen by experiments. Edge likelihood reflects the similarity of the changes in flame shape, spatial variation and spatial distribution; it can be used to distinguish the common interference targets, such as fast-moving fixed highlight, flame color moving objects, a large area of the Illumination changes and so on.

Sharp corner. Flame shape features in the video image is shown as an image with a multi-layer closed contour, there are one or more sharp corners on the contour line. Sharp corners must satisfy two conditions, first of all it should have a vertex, and then the value on both sides of it must be greater than a threshold L. if fire happened there must be a lot of sharp corners on the contour of the potential fire region, here set the minimum number is $\mathrm{N}_{\min }$.

Target Circularity. Circularity is a parameter to measure degree of the object like a circular shape or the area complexities. It is calculated by object area size and perimeter as follows:

$$
C=\frac{4 \pi S_{o}}{P^{2}}
$$

where $\mathrm{S}_{\mathrm{o}}$ is the area size of the target and $\mathrm{P}$ is the perimeter. In general, the fire flames have complex, irregular shape, while other similar fire interferences (such as cigarette, lights) have regular shapes with high circularity. If the potential target has a smaller circularity than $\mathrm{C}_{\mathrm{t}}$, it can be identified that there are a fire in the image. The extraction of sharp corners and calculation of target circularity are all Criterions based on the polygonal and irregular characters of flame.

\section{Fire Alarm System and Experimental Results}

Based on above analysis, an intelligent multi-stage decision strategy system is proposed. Time difference is firstly used to process the gray-scale image to obtain the moving region in the scene, then apply color segmentation to get the ROI of fire region, thirdly shape features such as randomness of area size, edge likelihood are calculated to avoid some interference, at last the polygonal and irregular characters of flame like sharp corners and circularity are used to identify the fire. 
The performance of algorithm is showed by following Figures: 6 image sequences were tested by the given fire detection method, mainly for forest and lights.
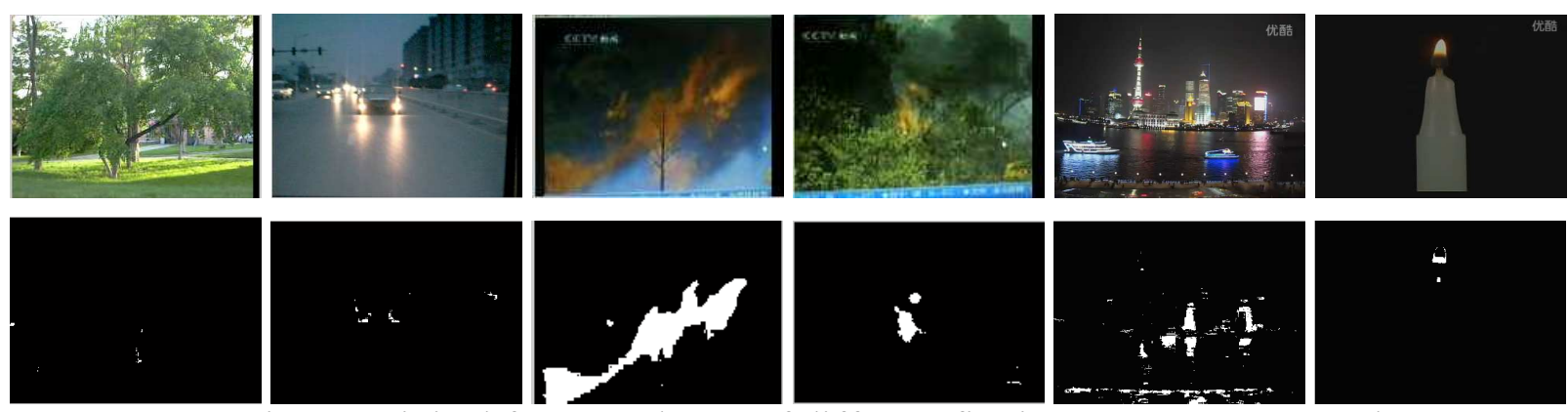

Fig. 1 Original frame and ROI of different fire image sequence samples

The upper line is random frames in the sequence and the lower ones are the ROI image after motion detection and color segment. The features and fire alarm result is showed in Tab.1.

Table 1 Fire alarm result

\begin{tabular}{|c|c|c|c|c|c|c|}
\hline & $\mathrm{g}_{\text {sum }}$ & $\nabla \mathrm{A}_{\mathrm{i}}$ & $\varepsilon_{i}$ & Sharp corner & Circularity & Fire alarm \\
\hline Threthod & $\mathrm{T}_{2=0.0015}$ & $\Lambda=0.0350$ & $\theta=0.7$ & $\mathrm{~N}_{\min =5}$ & $\mathrm{C}_{\mathrm{t}=0.55}$ & \\
\hline 1 & $4.5437 \mathrm{e}-004$ & - & - & - & - & No \\
\hline 2 & 0.0483 & 0.0175 & - & - & - & No \\
\hline 3 & 0.1833 & 0.0385 & 0.1128 & 22 & 0.3414 & Yes \\
\hline 4 & 0.1233 & 0.0901 & 0.0614 & 19 & 0.5234 & Yes \\
\hline 5 & 0.1517 & 0.0470 & 0.0250 & 34 & 0.5785 & No \\
\hline 6 & 0.0181 & 0.0886 & 0.7120 & - & - & No \\
\hline
\end{tabular}

Experimental result shows the Fire-detection method presented in this paper could detect fire in the image sequence effectively, and it is capable of distinguishing Environmental light changes, background color interference and light false identification. Multi-stage decision strategy can improve the algorithm performance and reduce false-alarm rate.

\section{Conclusion}

The proposed method has broad application prospects in the important military, social security, forest-fire alarm, commercial applications, and so on. Because of many thresholds must be adjust under different conditions, this multi-stage decision strategy has some shortcomings, in future learning method or fuzzy theory could be add into this fire alarm system, which should further improve the intelligence and adaptability of the system.

\section{References}

[1] zilawati Mohd Razmi, Nordin Saad, Vijanth Sagayan Asirvadam, Vision-Based Flame Detection,J.Motion Detection \& Fire Analysis, 2010, 13-16.

[2] B.U. To"reyin, Y.Dedeoglu, U.Gudukbay, A.E.Cetin, Computer vision based method for real-time fire and flame detection, J. Short Communication Pattern Recognition Letters, 2006,27(1)49-58.

[3] PauloViniciusKoerichBorges, EbroulIzquierdo, A Probabilistic Approach for Vision-Based Fire Detection in Videos, C. IEEE trans. Circuits and systems for viseo technology,2010,vol.20.

[4] Tung Xuan Truong, Yongmin Kim, Jongmyon Kim, Fire Detection in Video Using genetic-Based Neural Networks, International Conference on Information Science and Applications (ICISA), $2011,1-5$.

[5] Rafiee, A., Tavakoli, R., Dianat, R., Abbaspour, S., Jamshidi, M., Fire and Smoke Detection Using Wavelet Analysis and Disorder Characteristics, 3rd International Conference on Computer Research and Development (ICCRD), 2011, 262 - 265. 